\title{
REFLEXO CILIOESPINHAL NO HOMEM
}

\author{
Fernando Guilhon Henriques * \\ ITURIALDI AZEVEDO LEÃO **
}

A necessidade de reforçar o arsenal semiológico em lesões do tronco cerebral nos levou a estudar o reflexo cilioespinhal em pacientes com lesões nesta topografia. Este reflexo, estudado e descrito por Budge em 1852, consiste na dilatação pupilar rápida em resposta a estímulos nocivos cutâneos aplicados na face, região lateral do pescoço ou porção superior do torax.

O tamanho da pupila é regulado pela interação das vias parassimpática (constritora) e simpática (dilatadora). Assim, a dilatação pupilar pode depender tanto de uma inibição parassimpática quanto de uma excitação simpática. Qual dessas atividades (inibição, excitação) está relacionada com a midriase que se observa no reflexo cilioespinhal?

A via parassimpática começa no núcleo de Edinger-Westphal e segue pelo III nervo craniano alcançando o gânglio ciliar e, dai, o esfincter pupilar. A via simpática é ipsilateral e possui três neurônios: o primeiro, diencefálico, cujo axônio desce passando pelo mesencéfalo, ponte e bulbo, faz sinapse na coluna intermédio-lateral da medula torácica superior; os neurônios de segunda ordem saem com as três primeiras raizes torácicas, passam através do. gânglio estrelado e seguem o tronco simpático para fazerem sinapses no gânglio cervical superior; as fibras de terceira ordem acompanham a artéria carótida interna até o crânio e então passam através do rochedo para entrarem na cavidade craniana onde acompanham a divisão oftálmica do nervo trigêmio e seus ramos nasociliares até atingirem as fibras dilatadoras da pupila na iris (Fig. 1).

A via aferente do reflexo é constituída, na sua porção periférica, pelos ramos anteriores dos nervos espinhais (plexos cervical e braquial) ou pelos ramos do nervo trigêmio, conforme o estímulo seja aplicado no pescoço, torax ou na face. A porção central da via aferente deve corresponder ao feixe espinotalâmico.

Estudos experimentais em animais, bem como observações no homem têm suscitado discórdia quanto à predominância do sistema simpático ou do parassimpático mesencefálico, na regulação do reflexo cilioespinhal.

* Chefe da Unidade de Neurologia, 1.0 Hospital Distrital de Brasilia; ** Médíco Residente, Neurocirurgia. 
Weinstein e Bender ${ }^{8}$, em 1941, após estudos experimentais, afirmaram que no macaco a dilatação pupilar seria predominantemente devida a um aumento da atividade simpática enquanto que, no gato, seria decorrente de inibição parassimpática. Trabalhos semelhantes efetuados em animais por Jaffe (1949) ${ }^{4}$, Lowenstein e Lowenfeld (1950) ${ }^{5}$, levaram à conclusão de que há predomínio da excitação simpática. Arief e Pyzik (1953) ${ }^{1}$ verificaram, no homem, que a resposta depende inteiramente do sistema simpático, o que foi confirmado por Grossiord e col. (1961) ${ }^{2}$ e por Reeves e Posner (1969) 8 .

McNealy e Plum (1962) ${ }^{6}$ afirmaram que o reflexo cilioespinhal não é bloqueado por lesões preganglionares simpáticas cérvico-torácicas ou pelo bloqueio do gânglio estrelado; as lesões isoladas da porção lateral da medula que envolviam as vias simpáticas descendentes dilatadoras da pupila não bloquearam o reflexo. Estes autores admitem que a perda do reflexo cilioespinhal é indicadora de disfunção mesencefálica e concluíram afirmando que

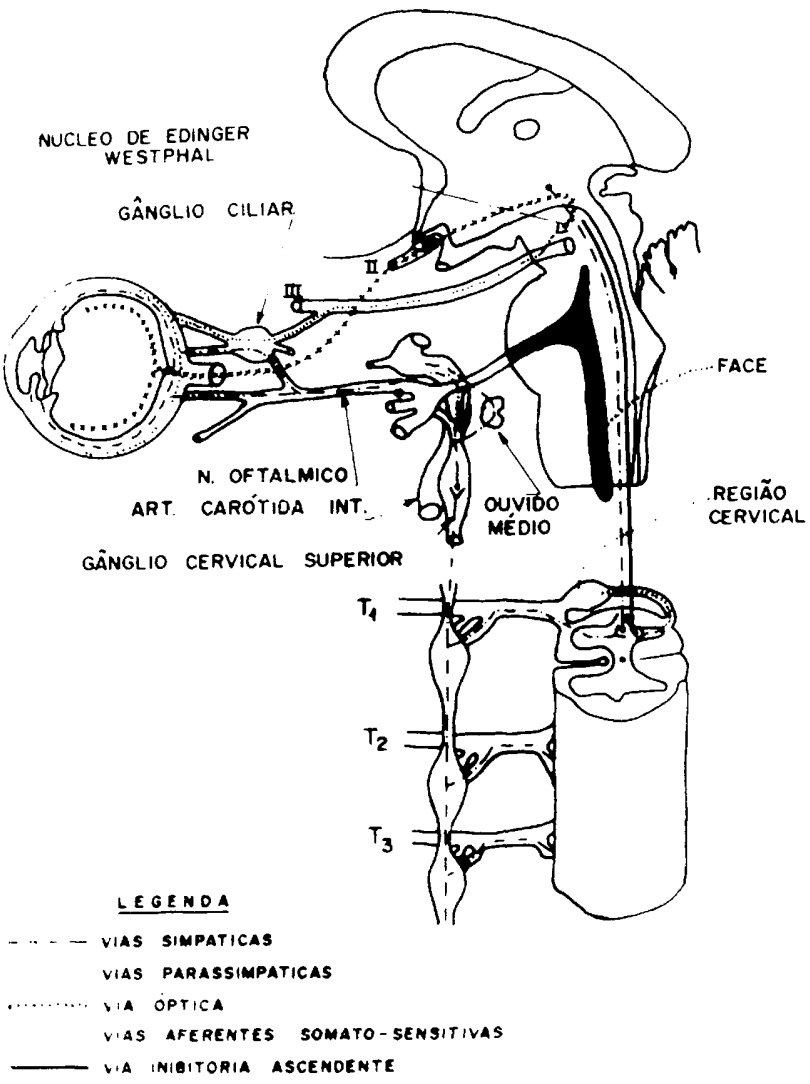

Fig. 1 - Sistemas aferentes e eferentes relacionados com o reflexo cilioespinhal. 
o reflexo é primariamente função de inibição parassimpática e que o sistema simpático pouco contribui, se o faz.

Harris, Hodes e Magoun (1944) ${ }^{3}$ já haviam demonstrado a existência de um sistema ascendente com funçōes inibitórias diretas sobre os núcleos de Edinger e Westphal no gato, cuja integridade seria necessária para que a dilatação pupilar reflexa ocorresse. Estes autores demonstraram ainda que esta via não corresponde ao feixe espinotalâmico como se poderia supor. Se é a inibição do parassimpático o fator fundamental para que a midríase reflexa ocorra, a integridade do tronco cerebral é importante para a obtenção da resposta reflexa, pois a via inibitória atravessa o tronco em sentido ascendente até o mesencéfalo ${ }^{3}$. O mesmo se poderia dizer do sistema simpático, muito embora este, por ser bastante extenso, pudesse manter a resposta do reflexo nas lesóes parciais.

\section{MATERIAL E METODO}

Foram estudados 23 pacientes, sendo 16 do sexo masculino e 7 do sexo feminino. A idade variou de 04 a 51 anos, com média de 29 anos. O reflexo cilioespinhal foi avaliado com auxilio de lupa após estímulo doloroso na face, região lateral do pescoço e face anterior do torax. Sua pesquisa ocorreu principalmente na fase aguda da lesão, evitando-se excesso de luminosidade; nos casos em que se tornava difícil a visualização da pupila, esta foi iluminada parcialmente com foco manual, o que não prejudicou a resposta pupilar. O reflexo foi avaliado quanto à sua presença ou ausência.

Os pacientes foram classificados em dois grupos: Grupo 1, que apresentavam lesão medular; Grupo 2, com lesão do tronco cerebral. Nos pacientes com lesão do tronco cerebral o diagnóstico foi firmado de acordo com critérios clínicos, em que foram analisados, principalmente, o nivel de consciência, a respiração, as pupilas, as respostas motoras e os movimentos oculares; os casos com lesão medular eram, em sua maioria, vítimas de traumatismo raque-medular com fratura e luxação em nível cérvico-torácico.

\section{RESULTADOS}

Dos 10 pacientes do grupo 1, cinco apresentavam déficit sensitivo motor nos quatro segmentos e disfunção esfincteriana; nestes casos o reflexo cilioespinhal se encontrava abolido bilateralmente. No caso 6 o paciente, homem de 47 anos, vitima de traumatismo raque-medular com fratura-luxação da primeira vértebra torácica encontrava-se paraplégico; o reflexo também se achava abolido bilateralmente, o que se poderia explicar pelo comprometimento do centro cilioespinhal de Budge. Nos três casos seguintes o reflexo se encontrava abolido apenas do lado em que o sistema simpático se encontrava comprometido: em dois deles havia sindrome de Claude Bernard-Horner completa e no terceiro apenas esboçada. No último caso, a paciente apresentava diparesia braquial atrófica, sendo constatada à cirurgia presença de hidromielia. Neste caso o reflexo cilioespinhal se encontrava abolido à esquerda, lado em que a sintomatologia era mais acentuada (Quadro 1).

Dos 13 pacientes do grupo 2, dois eram vitimas de acidente vascular cerebral (Quadro 5) e os demais tínham sofrido traumatismo cranioencefálico. No quadro 2 encontramos 5 casos, referentes aos pacientes mais graves, todos em coma profundo que evoluíram para o óbito. Apresentavam: distúrbios respiratórios graves ou evoluiram para os mesmos em fase final, reflexo fotomotor abolido bilateralmente, 


\begin{tabular}{llccc}
\hline Caso & $\begin{array}{c}\text { Idade } \\
(\text { anos })\end{array}$ & Sexo Quadro clínico & Reflexo cilioespinhal \\
& D. & E. \\
\hline
\end{tabular}

\begin{tabular}{|c|c|c|c|c|c|c|}
\hline \multirow{2}{*}{1} & 17 & M & tetraparesia & TRM & & \\
\hline & & & & luxação $C_{j}-C_{\varepsilon}$ & a. & a. \\
\hline \multirow[t]{2}{*}{2} & 20 & M & tetraplegia & $\mathrm{TRM}-\mathrm{C}_{\boldsymbol{\tau}}$ & & \\
\hline & & & & secção medular & a. & a. \\
\hline \multirow[t]{2}{*}{3} & 30 & M & tetraplegia & $\mathrm{TRM}-\mathrm{C}_{5}-\mathrm{C}_{6}$ & & \\
\hline & & & & fratura-luxação & a. & a. \\
\hline \multirow[t]{2}{*}{4} & 35 & $\mathbf{M}$ & tetraplegia & $\mathrm{TRM} \longrightarrow \mathrm{C}_{5}-\mathrm{C}_{6}$ & & \\
\hline & & & & fratura-luxação & a. & a. \\
\hline \multirow[t]{2}{*}{5} & 27 & $\mathbf{M}$ & tetraplegia & Tumor & & \\
\hline & & & & extramedular & a. & a. \\
\hline \multirow[t]{2}{*}{6} & 47 & M & paraplegia & $\mathrm{TRM}-\mathrm{T}_{1}$ & & \\
\hline & & & & fratura-luxação & a. & a. \\
\hline \multirow[t]{3}{*}{7} & 49 & $\mathrm{M}$ & paraplegia & Carcinoma & & \\
\hline & & & $\mathrm{CBH}$ à $\mathrm{E}$. & indiferenciado & p. & a. \\
\hline & & & & $\mathrm{C}_{7}-\mathrm{T}_{2}$ & & \\
\hline \multirow[t]{2}{*}{8} & 21 & F & monoplegia & Esclerose em & & \\
\hline & & & braquial à E. & placas & p. & a. \\
\hline \multirow[t]{2}{*}{9} & 20 & M & tetraplegia & $T R M-C_{1}-C_{5}-C_{6}$ & a. & p. \\
\hline & & & miose à D. & fratura-luxação & & \\
\hline \multirow[t]{3}{*}{10} & 37 & $\mathbf{F}$ & diparesia & Hidromielia & & \\
\hline & & & braquial mais & & p. & a. \\
\hline & & & intensa à $\mathrm{E}$. & & & \\
\hline
\end{tabular}

Quadro 1 - Reflexo cilioespinhal no homem: resposta pupilar nas lesões medulares. Legenda: $T R M=$ traumatismo raque medular; $C B H=$ Claude Bernard-Horner; $D_{.}=$direito; $E .=$ esquerdo; $a .=$ ausente; $p .=$ presente. 
sindrome de descerebracão. Nestes pacientes o reflexo cilioespinhal se encontrava abolido bilateralmente. No quadro 3 , encontramos os casos 6,7 e 8 relativos a pacientes com quadro clínico menos grave que o grupo anterior, nos quais o reflexo cilioespinhal se encontrava ausente apenas do lado da midriase fixa. Todos apresentavam síndrome de descerebração e distúrbios respiratórios de graves a moderados. No quadro 4 liguram os 3 casos de pacientes com lesões menos severas; no entanto todos estavam em coma, reagindo aos estimulos em postura de decorticação, caracterizando uma lesão em nivel mais alto e menos grave que a reação em descerebracāo. Apresentavam pupilas anisocóricas e reflexo fotomotor presente. Em dois casos a respiração era eupnêica e, no outro era irregular, caracterizando a hiperventilação neurogênica central na fase aguda, com melhora ulterior. Este último caso evoluiu para o óbito em virtude de insuficiência renal aguda desenvolvida pós-trauma. Em todos estes casos o reflexo cilioespinhal se encontrava presente bilateralmente.

No quadro 5 temos os dois últimos casos da série. Trata-se de pacientes lúcidos, com lesões vasculares ao nivel do tronco cerebral. O reflexo cilioespinhal se encontrava abolido do lado direito, correspondendo ao lado da síndrome de Claude Bernard-Horner em um caso e, do mesmo lado, no outro paciente com sindrome de Wallenberg, que apresentava miose do lado direito.

\begin{tabular}{|c|c|c|c|c|}
\hline Caso & $\begin{array}{l}\text { Idade } \\
\text { (anos) }\end{array}$ & Sexo & Quadro clínico & Diagnóstico \\
\hline
\end{tabular}

$151 \quad M \quad$ Coma. Hiperventilação neurogênica central. Pupilas isocóricas. Fotomotor ausente. Corneano presente. Descerebração.

$211 \quad M \quad$ Coma Respiração atáxica. Anisocoria com miose bilateral. Fotomotor ausente. Corneano ausente. Descedebração.

$3 \quad 35 \quad M$

$4 \quad 50 \quad M$

$\begin{array}{lll}5 & 47 & \text { M }\end{array}$

Coma. Respiração eupnêica. Anisocoria com midriase fixa à direita. Fotomotor ausente. Descerebração.

Coma. Respiração eupnêica. Miose bilateral. Fotomotor ausente. Corneano hiporreativo. Descerebração.

Coma. Hiperventilação neurogênica central. Anisocoria com midriase à esquerda. Fotomotor ausente. Descerebração.
Contusão cerebral. Hematoma subdural agudo à direita.

Contusão cerebral. Fratura de crânio.

Contusão cerebral. Hematoma subdural agudo à direita.

Contusão cerebral. Contusão de tronco cerebral.

Contusão laceração cerebral. Fratura de crânio.

Quadro 2 - Reflexo cilioespinhal no homem: resposta pupilar nas lesões do tronco cerebral. Nestes casos não houve resposta reflexa cilioespinhal. 


\begin{tabular}{|c|c|c|c|c|c|c|}
\hline \multirow[t]{2}{*}{ Caso } & \multirow[t]{2}{*}{$\begin{array}{l}\text { Idade } \\
\text { (anos) }\end{array}$} & \multirow[t]{2}{*}{ Sexo } & \multirow[t]{2}{*}{ Quadro clínico } & \multirow[t]{2}{*}{ Diagnóstico } & \multicolumn{2}{|c|}{$\begin{array}{c}\text { Reflexo } \\
\text { cilioespinhal }\end{array}$} \\
\hline & & & & & D. & E. \\
\hline 6 & 4 & M & $\begin{array}{l}\text { Coma. Respiracão eup- } \\
\text { nêica. Pupilas anisocóri- } \\
\text { cas com midriase à E. } \\
\text { Fotomotor à E. ausente. } \\
\text { Corneano presente. Des- } \\
\text { cerebração. }\end{array}$ & $\begin{array}{l}\text { Higroma subdu- } \\
\text { ral a E. Fra- } \\
\text { tura de cránio } \\
\text { com afundamen- } \\
\text { to. }\end{array}$ & p. & a. \\
\hline 7 & 25 & $\mathbf{F}$ & $\begin{array}{l}\text { Coma. Respiracão Chey- } \\
\text { ne Stokes. Pupilas aniso- } \\
\text { córicas com midriase à } \\
\text { E. Fotomotor hiporreativo. } \\
\text { Corneano presente. Des- } \\
\text { cerebração. }\end{array}$ & $\begin{array}{l}\text { Hematoma ex- } \\
\text { tradural esquer- } \\
\text { do. Fratura de } \\
\text { crânio. }\end{array}$ & p. & a. \\
\hline 8 & 25 & $\mathbf{M}$ & $\begin{array}{l}\text { Coma. Respiração Chey- } \\
\text { ne Stokes. Pupilas aniso- } \\
\text { córicas com midríase à E. } \\
\text { Corneano presente. Des- } \\
\text { cerebraçāo. }\end{array}$ & $\begin{array}{l}\text { Hematoma ex- } \\
\text { tradural esquer- } \\
\text { do. Fratura de } \\
\text { crânio. }\end{array}$ & p. & a. \\
\hline
\end{tabular}

Quadro 3 - Reflexo cilioespinhal no homem: resposta pupilar nas lesões do tronco cerebral. Legenda: $D .=$ direito; $E .=$ esquerdo; $p .=$ presente; $a$. = ausente.

\begin{tabular}{|c|c|c|c|c|}
\hline Caso & Idade & Sexo & Quadro clínico & Diagnóstico \\
\hline 9 & 19 & $F$ & $\begin{array}{l}\text { Coma. Respiração eupnêica. Pupilas } \\
\text { isocóricas. Fotomotor presente. Cor- } \\
\text { neano presente. Babinski bilateral. }\end{array}$ & Contusão \\
\hline 10 & 15 & $\mathbf{F}$ & $\begin{array}{l}\text { Coma. Respiração eupnêica. Pupilas } \\
\text { isocóricas. Fotomotor presente. Cor- } \\
\text { neano presente. Decorticação. }\end{array}$ & $\begin{array}{l}\text { Contusão cerebral. } \\
\text { Disfunção do tronco } \\
\text { cerebral. }\end{array}$ \\
\hline 11 & 23 & $\mathbf{M}$ & $\begin{array}{l}\text { Coma. Hiperventilação neurogênica } \\
\text { central. Pupilas isocóricas. Fotomo- } \\
\text { tor hiporreativo. Decorticação. }\end{array}$ & $\begin{array}{l}\text { Contusão cerebral. } \\
\text { Disfunção do tronco } \\
\text { cerebral. }\end{array}$ \\
\hline
\end{tabular}

Quadro 4 - Reflexo cilioespinhal no homem: resposta nas lesões do tronco cerebral. Nestes casos a resposta reflexa cilioespinhal se encontrava presente bilateralmente. 


\begin{tabular}{|c|c|c|c|c|c|c|}
\hline \multirow[t]{2}{*}{ Caso } & \multirow[t]{2}{*}{$\begin{array}{l}\text { Idade } \\
\text { (anos) }\end{array}$} & \multirow[t]{2}{*}{ Sexo } & \multirow[t]{2}{*}{ Quadro clínico } & \multirow[t]{2}{*}{ Diagnóstico } & \multicolumn{2}{|c|}{$\begin{array}{c}\text { Reflexo } \\
\text { cilioespinhal }\end{array}$} \\
\hline & & & & & D. & E. \\
\hline 12 & 33 & $\mathbf{F}$ & $\begin{array}{l}\text { Lúcida. Respiração eupnēica. } \\
\text { Pupilas anisocóricas com mio- } \\
\text { se à D. Fotomotor presente. } \\
\text { CBH à D. Função motora } \\
\text { normal. }\end{array}$ & $\begin{array}{l}\text { AVC bulbo } \\
\text { pontino à } D .\end{array}$ & a. & p. \\
\hline 13 & 30 & F & $\begin{array}{l}\text { Lúcida. Respiração eupnêica. } \\
\text { Anisocoria com miose à D. } \\
\text { Fotomotor presente. Cornea- } \\
\text { no abolido à direita. }\end{array}$ & $\begin{array}{l}\text { Sindrome de } \\
\text { Wallenberg }\end{array}$ & a. & p. \\
\hline
\end{tabular}

Quadro 5 - Reflexo cilioespinhal no homem: resposta pupilar nas lesōes do tronco cerebral. Legenda: $A V C=$ acidente vascular cerebral; $C B H=$ Claude Bernard-Horner; $D .=$ direito; $E .=$ esquerdo; $a .=$ ausente; $p=$ presente.

\section{COMENTARIOS}

Deste estudo torna-se evidente, observando-se os pacientes com lesão medular (Quadro 1), que a integridade do sistema simpático é fundamental para a existência do reflexo cilioespinhal: nestes casos não havia impedimento para que a resposta ocorresse por inibição parassimpática.

Com relação aos pacientes com lesão no tronco cerebral, se observarmos o grupo de doentes mais graves (Quadro 2), poderemos notar que havia sempre disfunção severa da regulação do diâmetro pupilar, ora com midriase unilateral, ora com miose bilateral, ou ainda, com anisocorias de vários tipos. Em todos os casos o reflexo estava abolido e isto poderia depender de fatores conjuntos que a nosso ver não podem ser interpretados.

Nos pacientes do quadro 3 , com lesão no tronco cerebral menos grave que o grupo anterior, observou-se midriase fixa unilateral. Concluimos que nesses pacientes o reflexo não foi observado deste lado porque a pupila não poderia se dilatar mais.

Nos pacientes incluídos no quadro 4, ambos os sistemas - parassimpático e simpático - pareciam preservados e o reflexo cilioespinhal estava presente. No entanto, novamente no grupo de pacientes do quadro 5, encontramos evidência de lesão simpática com abolição ipsilateral do reflexo.

Assim, pelo que conseguimos apurar, julgamos que a resposta do reflexo cilioespinhal no homem, é veiculada através do sistema simpático, em seus vários segmentos desde o tronco cerebral até a medula, e não conseguimos 
comprovar a idéia de fator inibitório parassimpático, importante para a existência da resposta reflexa, como querem Harris, Hodes e Magoun ${ }^{3}$, Mc Nealy e Plum ${ }^{6}$, entre outros.

\section{RESUMO}

Os autores estudaram o reflexo cilioespinhal em 23 pacientes, divididos em dois grupos: com lesão medular e com lesão no tronco cerebral. São discutidas as vias e o mecanismo do reflexo, bem como sua importância para o diagnóstico das lesões do tronco cerebral.

\section{SUMMARY}

\section{The ciliospinal reflex in man}

The study of the ciliospinal reflex in twenty-three patients is reported. The patients were divided into two groups: the first with spinal cord lesions and the second one with brain stem lesions. The authors discuss the pathways and the mechanisms of the ciliospinal reflex in man and emphasize their value on the diagnosis of brain stem lesions.

\section{REFERENCIAS}

1. ARIEFF, A. J. \& PYZIK, S. W. - The ciliospinal reflex in injuries of the spinal cord in man. Arch. Neurol. Psychiat. (Chicago) 70:621, 1953.

2. GROSSIORD, A.; HELD, J. P.; ODIEVRE, M. \& MARTIN, M. - Mydriase par excitation nociceptive souslésionnelle chez les tétraplégiques. Rev. Neurol. (Paris) 104:310, 1961.

3. HARRIS, A. J.; HODES, R. \& MAGOUN, H. W. - The afferent path of the pupillodilator reflex in the cat. J. Neurophysiol. 7:231, 1944.

4. JAFFE, N. S. - True psychosensory dilation and delayed psychosensory dilation of the pupil: a preliminary report. Amer. J. Ophthal. 32:1681, 1949.

5. LOWENSTEIN, O. \& LOWENFELD, I. E. - Role of sympathetic parasympathetic systems in reflex dilation of the pupil. Arch. Neurol. Psychiat. (Chicago) $64: 313,1950$

6. MCNEALY, D. E. \& PLUM, F. - Brainstem dysfunction with supratentorial mass lesions. Arch. Neurol. (Chicago) 7:26, 1962.

7. REEVES, A. G. \& POSNER, J. B. - The ciliospinal response in man. Neurology (Minneapolis) 19:1145, 1969.

8. WEINSTEIN, E. A. \& BENDER, M. B. - Pupillodilator reactions to sciatic and diencephalic stimulation: a comparative study in the cat and monkey. $J$. Neurophysiol. 4:44, 1941 .

Unidades de Neurologia e Neurocirurgia - 1." Hospital Distrital - 70000 Brasilia, D.F. - Brasil. 